\title{
Surgical repair of multiple muscular ventricular septal defects: The role of re-endocardialization strategy
}

Bahaaldin Alsoufi, MD, a,b Tara Karamlou, MD, ${ }^{a}$ Masaki Osaki, MD, ${ }^{a}$ Mitesh V. Badiwala, MD, ${ }^{a}$ Chan Chee Ching, MS, Ann Dipchand, MD, ${ }^{a}$ Brian W. McCrindle, MD, MPH, ${ }^{a}$ John G. Coles, MD, ${ }^{a}$ Christopher A. Caldarone, MD, ${ }^{a}$ William G. Williams, MD, and Glen S. Van Arsdell, MD ${ }^{a}$

Supplemental material is available online.
From the Cardiac Center, The Hospital for Sick Children, ${ }^{a}$ University of Toronto, Toronto, Ontario, Canada, and King Faisal Heart Institute, King Faisal Specialist Hospital and Research Centre, ${ }^{\mathrm{b}}$ Riyadh, Saudi Arabia.

Read at the Eighty-sixth Annual Meeting of The American Association for Thoracic Surgery, Philadelphia, Pa, April 29-May 3, 2006.

Received for publication May 12, 2006; revisions received July 11,2006 ; accepted for publication July 13, 2006.

Address for reprints: Glen S. Van Arsdell, MD, The Hospital for Sick Children, 555 University Ave, Toronto, Ontario, Canada, M5G 1X8 (E-mail: glen.vanarsdell@ sickkids.on.ca).

J Thorac Cardiovasc Surg 2006;132:1072-80 $0022-5223 / \$ 32.00$

Copyright (๑) 2006 by The American Association for Thoracic Surgery

doi:10.1016/j.jtcvs.2006.07.011
Objective: Surgical repair of multiple muscular ventricular septal defects (Swiss cheese septum) is associated with important morbidity and mortality. We sought to examine factors associated with permanent heart block, early mortality, and time-related survival. Additionally, we evaluated a new approach, transatrial re-endocardialization of interventricular septum, to mitigate risk.

Methods: One hundred sixteen patients underwent surgery for multiple muscular ventricular septal defects (1982-2005), of whom 64 (55\%) had associated cardiac anomalies. Twenty-seven consecutive patients (median age 0.54 years, range 15 days-7.2 years) underwent transatrial re-endocardialization (2002-2005). Forty-four percent had Swiss cheese septum ( $>4$ defects). Multivariable regression analysis determined risk factors for pacemaker and survival.

Results: Operative mortality for the entire cohort was $9 \%$. Risk factors for death were double-outlet right ventricle (odds ratio 44.7, $P=.003$ ), ventriculotomy (odds ratio $6.4, P=.03$ ), and fewer multiple muscular ventricular septal defects repaired (odds ratio 4.7/defect, $P=.04$ ). Era mortalities differed: $16 \%$ for 1982 through 1990, $13 \%$ for 1990 through 1998, and $0 \%$ for 1999 through 2005, $P=.006$ ). Fourteen patients (12\%) required a pacemaker. Time-related survivals at 1 and 10 years were $90 \% \pm 3 \%$ and $82 \% \pm 5 \%$. Risk factors for death were double-outlet right ventricle (hazard ratio 8.3, $P=.02$ ) and longer bypass (hazard ratio $1.02 / \mathrm{min}$, $P=.02)$. In 27 re-endocardialization patients, a combined closure strategy to close 184 defects were applied: transatrial re-endocardialization (median 5, range 2-21), patch (median 1, range 0-4), and device (range 0-1). Post-repair ventricular function was good in 25 of 27 patients. The median number of residual defects was 1.5 (range 0-3), and median residual jet width on color Doppler was $2.3 \mathrm{~mm}$ (range 0-4.2 mm). One child required a pacemaker. There were no early or late deaths.

Conclusions: Outcome of surgical repair of multiple muscular ventricular septal defects (Swiss cheese septum) has improved. Transatrial re-endocardialization strategy enables early complete or nearly complete obliteration of multiple muscular ventricular septal defects with minimal residual lesions (shunt, ventricular dysfunction). Long cardiopulmonary bypass duration is well tolerated. The incidence of permanent heart block has improved. Early echocardiographic and clinical outcomes are promising.

$\mathrm{S}$ urgical treatment of children with multiple muscular ventricular septal defects (mMVSDs) remains a challenge. It is associated with significant operative mortality and complications, including ventricular dysfunction and complete heart block. ${ }^{1-7}$ These lesions occur in isolation but also often exist in combination with other congenital cardiac lesions, such as transposition of the great arteries, double-outlet right ventricle, and coarctation of the aorta, further complicating the management of these children. ${ }^{1-7}$ 


\section{Abbreviations and Acronyms \\ $\mathrm{CPB}=$ cardiopulmonary bypass \\ DORV $=$ double-outlet right ventricle \\ MVSD $=$ muscular ventricular septal defect \\ mMVSDs $=$ multiple muscular ventricular septal defects \\ TAR = transatrial re-endocardialization \\ VSD $=$ ventricular septal defect}

Optimal management of mMVSDs remains controversial. Several techniques have been described, including initial pulmonary artery banding, ${ }^{4,6}$ single-stage repair with a transatrial or a transventricular approach, ${ }^{1-4,6-8}$ interventional transcatheter device closure, ${ }^{9}$ and a hybrid strategy with intraoperative patch or perventricular device closure techniques. ${ }^{5,10-14}$ Although different groups have achieved successful results with all these strategies, each technique is associated with important limitations.

In the last 4 years, we adopted a new treatment strategy: transatrial re-endocardialization (TAR). It is based on the concept that there are a finite number of trabeculations that can be primarily approximated for defect closure. At completion, the right ventricular endocardial cavity is smooth. Deep suturing, which may be a cause of ventricular dysfunction and heart block, is avoided.

In this study, we sought to examine surgical results in infants and children undergoing primary closure of mMVSDs through 23 years at our institution, including those requiring repair of associated lesions. We intended to define patient and procedural risk factors for early and late death, and for the development of complete heart block. In addition, we aimed to evaluate the early results of the TAR strategy and assess its role in addressing the major problems associated with surgical closure of mMVSDs.

\section{Patients and Methods}

Approval of this study was obtained from the research ethics board at The Hospital for Sick Children. From July 1982 to September 2005, a total of 133 children ( $<18$ years) with mMVSDs underwent surgical repair at our institution. The patients were identified by the surgical database. Clinical, operative, and outcome data were abstracted from the medical records. Patients who had a diagnosis of mMVSDs but did not undergo initial or subsequent closure of the defects were excluded. The risk factors for the remaining 116 patients were analyzed.

\section{Ventricular Septal Defect Characteristics}

A muscular ventricular septal defect (MVSD) is defined as an intraventricular communication that has a rim consisting entirely of muscle. We used the ventricular septal defect (VSD) classification of Van Praagh and Kirklin. ${ }^{1,15}$ Posterior muscular defects are located in the inlet area (infratricuspid or inlet septal) of the muscular septum, anterior muscular defects are located anterior to the septal band of the septomarginal trabeculation, midtrabecular defects are proximal to the moderator band, and apical defects are below the moderator band in the ventricular apex.

A variety of descriptions have been used to define the highly deficient ventricular septum with uncountable number of muscular defects (Swiss cheese septum). The recent congenital heart surgery nomenclature defines Swiss cheese septum as 4 or more MVSDs. ${ }^{15}$

MVSDs in each patient were characterized by preoperative echocardiographic imaging and intraoperative inspection. It has been our experience, however, that echocardiography often underestimates the number of mMVSDs and that intraoperative exploration frequently reveals more defects that are detected by probing the interventricular septum with a blunt-tip instrument.

\section{Operative Technique of TAR Strategy}

Cardiopulmonary bypass (CPB) was established with mild hypothermia $\left(32^{\circ} \mathrm{C}-34^{\circ} \mathrm{C}\right)$ by standard aortic and bicaval venous cannulation. The left ventricle was decompressed by a vent placed through the interatrial septum. Myocardial protection was antegrade cold blood cardioplegia. In patients with associated cardiac abnormalities, the CPB technique was modified as necessary to allow correction of additional anomalies. If arch reconstruction was necessary, deep hypothermic circulatory arrest with profound hypothermia (with regional cerebral perfusion) was used. Exposure of the mMVSDs was done through a right atriotomy and through the tricuspid valve leaflets. It was possible to expose most of the mMVSDs, with the apical defects being the most difficult, followed by the anterior defects. Anterior defect exposure was sometimes facilitated by a transverse pulmonary arteriotomy. A few were identified from the left side through an atrial septal defect and the mitral valve. Identification of mMVSDs was done by gentle probing of trabeculations with a right angle clamp to delineate a connection with the left ventricle. Certain maneuvers, such as probing through a perimembranous VSD or through the interatrial septum, may be helpful to completely identify all mMVSDs. Occasionally, division of minor trabeculae (1-2 $\mathrm{mm}$ in diameter) was needed to delineate the edges of the defects or expose some apical defects. No major trabeculae or moderator band division was performed in any of the patients undergoing TAR. A ventriculotomy was not used to access defects unless it was required for other repair (eg, right ventricular-pulmonary arterial conduit).

Although real-time imaging on a beating heart is a useful tool for intraoperative localization of isolated big MVSDs, mMVSDs are notoriously difficult to identify before the initial repair. Initial echocardiography may reveal significant jets through large defects; once major defects such as large MVSDs or perimembranous VSDs are closed, however, additional small or moderately sized anterior and midtrabecular defects are unmasked and detected on postoperative echocardiography, thus necessitating the probing strategy.

The TAR strategy used 6-0 or 7-0 polypropylene sutures with a BV1 or BV175 needle (Ethicon, Inc, Somerville, NJ). The septal trabeculations were approximated in two layers of superficial, endocardial running sutures. Each suture was started at the distal edge of a trabeculation and run in a double-layer, simple, continuous fashion to the other end of the trabeculation, where they were tied (Figure 1). It was sometimes possible to close multiple trabeculations with the same suture. The TAR sutures were placed in all 


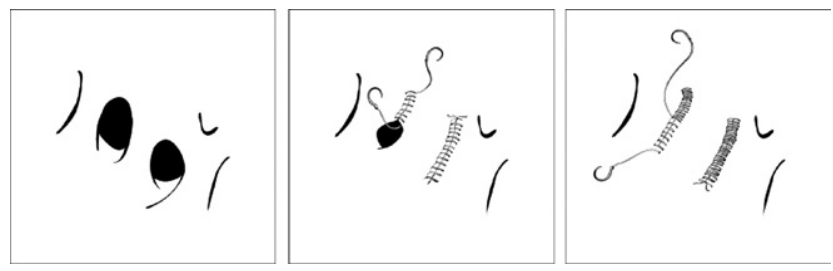

Figure 1. Re-endocardialization strategy relies on double-layer suturing of septal trabeculations to each other with fine, superficial, endocardial running sutures.

four compartments of the muscular septum (midtrabecular $\mathrm{n}=$ $18 / 27$, anterior $n=15 / 27$, apical $n=13 / 27$, and inlet/posterior $\mathrm{n}=8 / 27)$.

A combination of different techniques was used to achieve complete obliteration of the VSDs. Large MVSDs in the inlet/ posterior muscular septum were usually closed with a synthetic Dacron polyester fabric patch. Similarly, large apical MVSDs, especially those located just underneath the moderator band with a poorly defined muscular rim, were considered suitable for device closure. The technique of intraoperative device closure of MVSDs has been previously described. ${ }^{5}$ Figures 2 and 3 illustrate examples of our combined closure strategy to achieve complete obliteration of the intraventricular communications through the septum.

Evaluation of repair was by intraoperative transesophageal echocardiography. Patients with echocardiographic demonstration of potential significant residual shunt $(n=4 / 27)$ underwent intraoperative assessment of the shunt fraction. Complete operative data for patients undergoing the TAR strategy are presented in Table 1.

\section{Echocardiography and Follow-up}

Preoperative echocardiographic reports were collected and reviewed. The numbers and locations of mMVSDs were gathered. Postoperative echocardiograms for all patients undergoing TAR, usually done before hospital discharge, were retrospectively re-
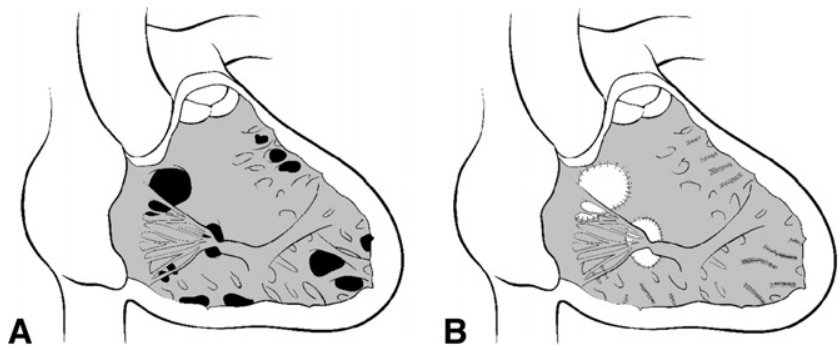

Figure 2. A, Preoperative example of patient 1 with multiple ventricular septal defects, including perimembranous defect, and multiple muscular defects involving posterior/inlet, midtrabecular, apical, and anterior compartments. B, Complete obliteration of ventricular septum was achieved in patient 1 with patch repair of perimembranous and large inlet muscular defects, with re-endocardialization sutures to obliterate completely remaining defects.
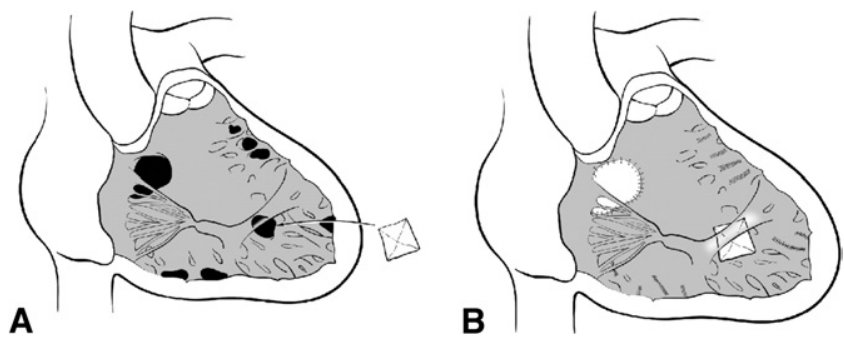

Figure 3. A, Preoperative example of patient 2 with multiple ventricular septal defects, including perimembranous defect, and multiple muscular defects involving posterior/inlet, midtrabecular, apical, and anterior compartments. B, Complete obliteration of ventricular septum was achieved in patient 2 with patch repair of perimembranous defect, intraoperative device closure of large apical defect underneath moderator band, and re-endocardialization sutures to obliterate completely remaining defects.

viewed by a single, blinded cardiologist (M.O.). The numbers and locations of residual shunts were reported. Additionally, the size of each residual defect was measured by color flow Doppler.

TABLE 1. Operative data for 27 patients with multiple muscular ventricular septal defects (mMVSDs) undergoing transatrial re-endocardialization (TAR)

\begin{tabular}{lc}
\hline No. of patients & 27 \\
No. of closed defects (median and range) & $7(3-22)$ \\
TAR & $5(2-21)$ \\
Patch & $1(0-4)$ \\
Device & $1(0-1)$ \\
Locations of TAR suture placement (No.)* & \\
Midtrabecular & 18 \\
Anterior & 15 \\
Apical & 13 \\
Inlet/posterior & 8 \\
Additional procedures (No.) & \\
Closure of perimembranous VSD† & 7 \\
Tricuspid valve repair & 6 \\
Aortic arch reconstruction/coarctation repair & 5 \\
Atrioventricular septal defect repair & 3 \\
Tetralogy of Fallot repair & 3 \\
Arterial switch & 1 \\
Unifocalization and RV-PA conduit & 1 \\
CPB (min, mean \pm SD) & $146 \pm 52$ \\
Isolated mMVSDs & $126 \pm 50$ \\
With associated lesions & $167 \pm 57$ \\
Aortic cross clamp time (min, mean \pm SD) & $112 \pm 38$ \\
Isolated mMVSDs & $104 \pm 40$ \\
With associated lesions & $122 \pm 34$ \\
\hline
\end{tabular}

VSD, Ventricular septal defect; RV-PA conduit, right ventricular-pulmonary arterial conduit. *Numbers indicate number of patients requiring TAR suturing in each compartment and not the total number of TAR closure sutures. †Closure of mMVSDs associated with only perimembranous VSD patch was still considered in the isolated mMVSDs group. 
Late outcomes were determined from clinic records when available or from written correspondence with patient physicians, which is continuously updated in our surgical database. Median follow-up time was 3.78 years (range 3 days-20.1 years).

For the TAR group, follow-up echocardiograms were performed at a median of 1 month (range 2 days-1.9 years) after repair. For the non-TAR group, follow-up echocardiograms were performed at a median interval of 4.1 months (range 1 day-6.3 years) after repair.

\section{Statistical Analysis}

Data are given as frequency, median with range, or mean $\pm \mathrm{SD}$ as appropriate, with the number of nonmissing values indicated. All data analyses were performed with SAS statistical software (version 9.1; SAS Institute, Inc, Cary, NC).

Overall cohort analysis $(n=116)$. Multivariable factors associated with the binary outcomes of pacemaker implantation and operative mortality (defined as death within 30 days of operation or at any time during the same hospital admission) were modeled with logistic regression. Multivariable factors associated with the number of residual VSDs after operation were modeled with linear regression. Time-related overall mortality after repair was initially evaluated with the Kaplan-Meier method, with risk-unadjusted comparisons between stratified groups investigated with the Gehan-Wilcoxon statistic because most events occurred early. Risk factors for time-related mortality were then sought with multivariable Cox proportional hazard models, and risk-adjusted survival was compared between groups. Variables were prescreened to prevent model overdetermination, with only those factors associated with more than five events used in the multivariable model. Multicollinearity among variables within linear regression models among variables was avoided by verifying that the variance inflation factor remained less than 2 for the full models. Variable transforms were applied to improve calibration with the risk scale, and interactions were tested among significant main effect terms in the full model. Score selection was used to select the best three candidate models, followed by a stepwise selection algorithm with an entry criterion of $P=.15$.

Propensity-adjusted comparison with contemporaneous patients $(\boldsymbol{n}=56)$. Because patients undergoing TAR $(\mathrm{n}=27)$ were far fewer and not contemporaneous with those undergoing other surgical repairs $(n=106)$, we next selected 29 of the most recently repaired non-TAR patients to form a comparison group of similar size operated on within our same era designation (era 3) as those undergoing TAR. This gave us a sample size of 56, of whom 27 underwent TAR and 29 underwent conventional repair of multiple VSDs. In comparing outcomes between these groups, a propensity score was created from multiple logistic regression, incorporating differences in demographic, morphologic, and procedural characteristics into a model that provided a probability that a given patient belonged to one group versus another (Table E1). This variable was then entered into the logistic model for need for pacemaker implantation after VSD repair and linear regression models for the number of residual VSDs and the cumulative size (in millimeters) of the residual VSDs, to attempt to adjust for differences in characteristics between the two groups.

\section{Results}

\section{Entire Patient Cohort}

The patient cohort included 116 children. Median age at time of the operation was 9 months (range 3 days-18.1 years). Fifty-two patients (45\%) had isolated mMVSDs, whereas $64(55 \%)$ had associated cardiac anomalies, such as coarctation of the aorta $(n=25)$, transposition of the great arteries $(\mathrm{n}=13)$, atrioventricular septal defect $(\mathrm{n}=8)$, tetralogy of Fallot $(\mathrm{n}=7)$, and other cardiac anomalies $(\mathrm{n}=$ 11). Forty-six patients $(40 \%)$ had previous pulmonary arterial banding. The complete clinical profile of the entire cohort is summarized in Table E2.

Early results for entire cohort. The median number of VSDs repaired was 3 (range 1-22). The mean aortic crossclamp time was $105 \pm 36$ minutes, and the mean CPB time was $149 \pm 52$ minutes.

Significant differences between patients undergoing TAR and those having conventional approaches were evident. The mean numbers of defects closed for those undergoing TAR versus conventional surgical repair were $6.8 \pm 3.8$ and $2.3 \pm 1.0$, respectively $(P<.001)$. The age at repair was younger in the TAR group than in those having conventional repair (median 0.54 years, range 0.04-7.24 years, vs median 1.07 years, range 3 days-18.1 years, $P=.005)$. CPB and aortic crossclamp times appeared to be longer in the TAR group than in the others, but statistical significance was not achieved.

The operative mortality was 10 of $116(9 \%)$. The univariable analysis of risk factors for operative mortality is listed in Table 2. Multivariable logistic regression model for operative mortality revealed the following significant independent factors: double-outlet right ventricle (DORV, odds ratio $44.7, P=.003$ ), ventriculotomy (odds ratio $6.4, P=$ .03 ), and smaller number of VSDs repaired (odds ratio 4.7 per VSD, $P=.04$; Table E3).

The incidence of complete heart block requiring placement of a permanent pacemaker was 14 of 116 (12\%). The univariable analysis of risk factors for pacemaker placement is listed in Table 2. Multivariable logistic regression model for pacemaker implantation revealed the following significant risk factors: DORV (odds ratio 9.7, $P=.03$ ) and postoperative pulmonary arterial banding (odds ratio 6.8, $P=.055$; Table E3).

Late results for entire cohort. Kaplan-Meier-estimated survivals from repair were $93 \% \pm 3 \%$ at 30 days, $90 \% \pm$ $3 \%$ at 1 year, and $82 \% \pm 5 \%$ at 7.4 years (Figure E1). Cox proportional hazard regression model for time-related death revealed the following risk factors: DORV (hazard ratio 8.27, confidence interval 1.4-49.3, $P=.02$ ) and longer CPB time (hazard ratio $1.02 / \mathrm{min}$, confidence interval 1.0-1.03, $P=.02)$.

Of importance, there was a significant interaction term between the length of $\mathrm{CPB}$ and era 3 , indicating that dura- 
TABLE 2. Univariable statistics (Fisher or $\left.\Pi^{2}\right)$ for operative mortality $(n=10 / 116)$ and pacemaker implantation $(n=14 / 116)$ for the entire cohort of patients with multiple muscular ventricular septal defects

\begin{tabular}{lcccr}
\hline Variable & No. dead & $\boldsymbol{P}$ value & No. of pacemakers & $\boldsymbol{P}$ value \\
\hline Re-endocardialization & 0 & .11 & $1(1 / 27,4 \%)$ & .18 \\
Era 1 (1982-1989) & $5(5 / 31,16 \%)$ & .12 & $6(5 / 31,16 \%)$ & .52 \\
Era 2 (1990-1998) & $5(5 / 39,13 \%)$ & .30 & $6(6 / 39,15 \%)$ & .55 \\
Era 3 (1999-2005) & 0 & .006 & $3(3 / 46,7 \%)$ & .16 \\
Ventriculotomy & $3(3 / 16,19 \%)$ & .12 & $3(3 / 16,19 \%)$ & .39 \\
Atrial repair & $7(7 / 98,7 \%)$ & .18 & $11(11 / 98,11 \%)$ & .46 \\
Any associated lesion & $8(8 / 64,13 \%)$ & .18 & $9(9 / 64,14 \%)$ & .57 \\
$\quad$ Atrioventricular septal defect & $1(1 / 8,13 \%)$ & .53 & $1(1 / 8,13 \%)$ & $<0.001$ \\
Truncus arteriosus & $1(1 / 2,50 \%)$ & .17 & 0 & $<0.001$ \\
Coarctation of aorta & $2(2 / 25,8 \%)$ & 00.001 & $3(3 / 25,12 \%)$ & $<0.001$ \\
Tetralogy of Fallot & 0 & $<0.001$ & $1(1 / 7,14 \%)$ & $<0.001$ \\
Double-outlet right ventricle & $2(2 / 4,50 \%)$ & .04 & $2(2 / 4,50 \%)$ & .07 \\
$\quad$ Transposition of great arteries & $2(2 / 13,15 \%)$ & .31 & $2(2 / 13,15 \%)$ & .66 \\
Previous pulmonary arterial band & $4(4 / 46,9 \%)$ & $<0.001$ & $7(7 / 46,15 \%)$ & .40 \\
Postoperative pulmonary arterial band & $1(1 / 5,20 \%)$ & .37 & $2(2 / 5,40 \%)$ & .11 \\
Younger age at operation & & .64 & & .55 \\
Longer cardiopulmonary bypass time & & .37 & & .57 \\
Longer aortic crossclamp time & & .71 & & .24 \\
Smaller number of defects repaired & & .14 & & .80 \\
\hline
\end{tabular}

tion of CPB has been neutralized in the more recent era. Risk-adjusted survival stratified by era of operation (era 3 vs others), shown in Figure E2, demonstrates that equivalent duration of CPB time was a risk factor in earlier eras but not within the most recent era (era 3; Table E3).

\section{TAR Group}

The most recent 27 consecutive children underwent the TAR procedure. Fourteen had isolated mMVSDs and 13 had associated cardiac defects, such as coarctation of the aorta $(\mathrm{n}=5)$, tetralogy of Fallot $(\mathrm{n}=3)$, atrioventricular septal defect $(n=3)$, and other anomalies $(n=2)$. Twelve patients $(44 \%)$ had more than 4 muscular defects (Swiss cheese septum) identified on preoperative echocardiography. These defects were apical $(31 \%)$, anterior $(30 \%)$, midtrabecular (24\%), and posterior/inlet muscular (16\%). The patient profile is summarized in Table E4.

The median number of MVSDs closed was 7 (range 3-22). Each patient had combined closure strategies as needed: TAR (median 5, range 2-21), patch (median 1, range 0-4), and device closure (range 0-1). Mean CPB and cardiac ischemic (crossclamp) times were $146 \pm 52$ and $112 \pm 38$ minutes, respectively. CPB and crossclamp times were longer for patients with associated defects than for those with isolated mMVSDs (CPB $167 \pm 57$ minutes vs $126 \pm 40$ minutes, $P=.04$, and crossclamp $122 \pm 34$ vs $104 \pm 40, P=.21$ ).

One patient required intraoperative revision for residual VSD around a device. None of the patients had postoperative pulmonary artery banding. Ventricular function on pos- trepair transesophageal echocardiography was normal or mildly reduced in 25 of 27 patients and moderately or severely reduced in 1 patient each. The median number of residual VSDs by color flow Doppler was 1.5 (range 0-3), and the median residual jet width was $2.3 \mathrm{~mm}$ (range $0-4.2$ $\mathrm{mm}$ ). One patient had an elevated shunt fraction of 2.1. One patient $(4 \%)$ required a permanent pacemaker for complete heart block. Median intensive care unit stay was 3 days (range 1-16 days). There was no operative or late mortality in the TAR group.

\section{Propensity-adjusted Comparison}

Pacemaker implantation. When pacemaker implantation was modeled with the addition of the propensity score, later year of operation was significant for lessened risk of pacemaker implantation (odds ratio 1.5/year since 1997, 95\% CI 1.02-2.36, $P=.04$ ).

Larger postoperative VSD size (as cumulative total of all residual VSDs). With a linear regression, propensityadjusted model, there was a trend toward a smaller cumulative VSD size on postoperative echocardiography for the TAR group $(P=.10)$. Other significant predictors were surgeon $(P=.03)$ and diagnosis of complete atrioventricular septal defects $(P=.04)$.

Number of residual VSDs. There were 32 patients who had 2 or more VSDs on their postoperative echocardiogram identified by color Doppler; 13 of these occurred in the TAR group, and the balance occurred in the non-TAR group. According to univariable statistics, there was no significant 
relationship between group and this outcome $(P=.29)$. With a logistic regression, propensity score-adjusted model, the presence of an anterior VSD compared with all other morphologic VSD types (odds ratio $6.5,95 \%$ confidence interval 1.4-28.4, $P=.01$ ) and smaller total VSD size on preoperative echocardiography (odds ratio $1.2 / \mathrm{mm}$ decrease, $95 \%$ confidence interval 1.0-1., $P=.04$ ) were significant.

\section{Discussion}

Ideal treatment for children with mMVSDs would be to achieve single-stage repair, completely obliterate the VSDs, and avoid heart block, arrhythmias, and ventricular dysfunction. The morbidity and mortality associated with treatment of mMVSDs remains higher than for many other diagnostic categories of congenital heart surgery. ${ }^{1-7}$ Several innovative approaches have been described, especially for those with a Swiss cheese septum. The multiplicity of management strategies is evidence that an effective treatment strategy to achieve all the ideal goals has not been reached.

The efficacy of any surgical approach to mMVSDs should be evaluated in terms of early operative risk, as well as septal function and rhythm status. Our hypothesis for the TAR strategy was that complete obliteration of mMVSDs could be achieved, along with avoidance of atrioventricular heart block and preservation of ventricular function, thereby diminishing mortality. Analysis of the data yields some interesting findings with respect to our hypothesis.

\section{Complete Obliteration of VSDs and Avoidance of Postoperative Shunts}

Failure to completely septate the ventricles and obliterate all defects may result in a left-right shunt associated with the need for placement of a pulmonary arterial band and a high rate of reoperation. ${ }^{1-6,10}$ From early experience in our overall cohort of patients, 3 of 6 patients who required postoperative pulmonary arterial banding for a residual shunt died. Our current analysis revealed that a smaller number of MVSDs closed was an independent factor for mortality on multivariable analysis. In the TAR group, our strategy was to close all exit points of interventricular communications on the right ventricular side. We frequently discovered more probe-detected defects than identified by preoperative echocardiography. The total number of right-sided probe-patent exit defects closed in 27 patients was 184, with a median of 7 (range 3-22). The anterior, midtrabecular, and inlet/ posterior defects at the diaphragmatic crux were easier to find, whereas identification of the apical defects was the most challenging. The median number of residual defects in all 27 patients undergoing the TAR strategy was 1.5 (range $0-3$ ) most were trivial, and many were not seen by 2-dimensional echocardiography (they would only be evident with color flow Doppler). The locations of residual shunts were in the midtrabecular and apical septum in 51\% and $24 \%$ of cases, respectively. Peridevice or peripatch leakage was seen in $14 \%$ of cases. The TAR strategy may prove to be particularly helpful in those patients with distal apical and anterior defects, where device application is difficult or impossible because of inadequate muscular rim. Although the exposure for apical defects may be challenging, the residual shunts have been tolerated.

\section{Preservation of Ventricular Function}

Surgical closure of mMVSDs is frequently complicated by postoperative ventricular dysfunction, thus complicating postoperative care of those patients and contributing to their morbidity and mortality. Several factors may play a role in postoperative ventricular dysfunction and hence are avoided in our current TAR strategy: (1) Division of the moderator band or thick trabeculae of the right ventricular septum to improve exposure and identification of defects is avoided. ${ }^{3,6}$ We have concerns regarding the effects of such divisions on the function of the right ventricle, and only minor 1- to 2-mm bands are occasionally divided in our practice. (2) Ventriculotomy is not performed to allow access to apical defects. ${ }^{2,3,8}$ There have been several reports of detrimental sequelae of left ventriculotomy, such as ventricular dysfunction, local apical aneurysm formation, late arrhythmias, and increased operative mortality. ${ }^{4,8,16}$ Our current analysis revealed that ventriculotomy was an independent factor for mortality on multivariable analysis. With our TAR strategy, we currently would not perform ventriculotomy for exposure of any MVSD. If a ventriculotomy is needed for the repair of associated cardiac lesions, such as a right ventricle to pulmonary artery conduit, however, placement of TAR sutures can be facilitated through that incision. Moreover, other approaches, such as gentle probing through the interatrial septum and exposure of the anterior defects through the pulmonary valve, can be used to facilitate exposure and intraoperative identification and closure of MVSDs. Although it has not been encountered in our series, we believe that exposure may be severely compromised in patients with dysplastic tricuspid valve and a small annulus, so this technique may not be suitable in such a patient. (3) Septal dysfunction as a result of deep suturing in the septum, division of major trabeculae, or application of multiple devices is avoided. The TAR strategy uses fine superficial sutures to approximate the trabeculae and eliminate exit defects on the right side of the septum. Two of 27 patients in the TAR series had moderate or severe dysfunction by immediate postoperative echocardiography. The patient with severe dysfunction had deeper sutures placed than is our current practice, whereas the other patient with moderate dysfunction had prolonged cardiac ischemic times and 22 defects closed. He had good recovery with normal biventricular function on later echocardiography. 


\section{Avoidance of Heart Block}

Surgical treatment of mMVSDs is associated with high incidences of septal dysfunction and complete heart block. ${ }^{1-7}$ Deep suturing or extending the sutures into the left side of the septum is associated with heart block. The incidence of complete heart block in the literature has been disturbingly high, even in cases in which device or double-patch hybrid closure techniques have been used. ${ }^{1-7,10}$ We avoid deep suturing and approximate the adjacent trabeculae with fine superficial sutures. One patient in the TAR series required a pacemaker. In this patient, sutures were placed into the left anterior septum (likely a technical issue). The low incidence of permanent heart block in the TAR group (4\%) is encouraging and compares favorably with the non-TAR group $(15 \%)$. The relatively small sample size and the different variables associated with those patients may have precluded meaningful analysis of the effect of TAR on postoperative heart block.

\section{Diminished Operative Mortality}

Surgical treatment of mMVSDs has been associated with elevated mortality. ${ }^{1-7}$ The presence of mMVSDs has been found to be an independent risk factor for early mortality after repair of complex congenital heart lesions, such as DORV or transposition of the great arteries. ${ }^{7,17}$ Similarly, we reported in a past publication that patients with mMVSDs with associated cardiac defects have an increased operative mortality risk. ${ }^{5}$

In our current analysis, ventriculotomy and smaller number of VSDs closed were independent factors for operative mortality. We consequently concluded that patients with residual lesions such as residual shunt, ventricular dysfunction, and arrhythmias are at higher risk for increased mortality. Moreover, longer CPB duration was found to be a significant factor for diminished overall survival for the entire patient cohort. Interestingly, the effect of longer CPB time has been neutralized as a risk factor for death in the current era. The TAR strategy, especially when associated with repair of additional cardiac anomalies, requires long $\mathrm{CPB}$ and cardiac ischemic times. Despite that, there were no early or late deaths in this group of patients. The cause of diminished mortality may be multifactorial. We hypothesize that the adverse effects of prolonged CPB and ischemic times are moderated by the fact that those children have minimal residual shunts, few arrhythmias, and little septal and ventricular dysfunction.

In our current practice, we recommend single-stage closure of mMVSDs at time of repair of associated cardiac anomalies or after the development of symptoms of heart failure in patients with isolated defects. We rarely use pulmonary artery banding because it is associated with multiple drawbacks, carries the inevitable need for reoperation, and may complicate subsequent repair. We would rarely reserve pulmonary artery banding for extremely ill children, such as those with cerebral bleeding, infection, or significant pneumonitis.

The TAR technique is a useful tool that can be applied as needed by surgeons, in addition to patch suturing or device application, in a combined closure strategy aiming to achieve complete obliteration of the interventricular communications. It is a safe adjunct that allows preservation of septal function and avoidance of heart block and has a special utility in Swiss cheese, apical, and anterior defects.

There are limitations of this technique. Premature infants and those with small tricuspid valve annuli are not good candidates, because exposure through the tricuspid valve is difficult in those cases. Although our results justify prolonged operative time and long CPB duration as needed to ensure complete repair and no residual pathology, patients who will have residual postoperative lesions that cannot be corrected or addressed by the surgeons at the time of repair (such as those with ventricular hypertrophy or significant pulmonary hypertension) will not tolerate prolonged ischemic time and extensive surgical repair.

\section{Conclusions}

The outcome of surgical repair of mMVSDs (Swiss cheese septum) has improved. An inherent risk of mortality is present in patients with mMVSDs, especially those with associated cardiac lesions that increase the complexity of the physiology and the surgical repair. A strategy that negates or diminishes the residual lesions largely mitigates risk. The technique of TAR enables complete or near complete obliteration of the Swiss cheese defects with preservation of the ventricular function in the vast majority of cases. Heart block is likely a technical complication and may be avoidable. The risk of mMVSDs and associated lesions has been largely mitigated. Early echocardiographic and clinical outcomes of TAR are promising. Future followup is needed to confirm the favorable results.

\section{References}

1. Kirklin JK, Castaneda AR, Keane JF, Fellows KE, Norwood WI Surgical management of multiple ventricular septal defects. J Thorac Cardiovasc Surg. 1980;80:485-93.

2. Wollenek G, Wyse R, Sullivan I, Elliott M, de Leval M, Stark J. Closure of muscular ventricular septal defects through a left ventriculotomy. Eur J Cardiothorac Surg. 1996;10(8):595-8.

3. Kitagawa T, Durham LA 3rd, Mosca RS, Bove EL. Techniques and results in the management of multiple ventricular septal defects. J Thorac Cardiovasc Surg. 1998;115:848-56.

4. Serraf A, Lacour-Gayet F, Bruniaux J, Ouaknine R, Losay J, Petit J, et al. Surgical management of isolated multiple ventricular septal defects. Logical approach in 130 cases. J Thorac Cardiovasc Surg. 1992;103:437-42.

5. Konstantinov IE, Coles JG. The role of intraoperative device closure in the management of muscular ventricular septal defects. Semin Thorac Cardiovasc Surg Pediatr Card Surg Annu. 2003;6:84-9.

6. Seddio F, Reddy VM, McElhinney DB, Tworetzky W, Silverman NH, Hanley FL. Multiple ventricular septal defects: how and when should they be repaired? J Thorac Cardiovasc Surg. 1999;117:134-9. 
7. Belli E, Lacour-Gayet F, Serraf A, Alkhulaifi AM, Touchot A, Bruniaux J, et al. Surgical management of transposition of great arteries associated with multiple ventricular septal defects. Eur J Cardiothorac Surg. 1999;16:14-20.

8. Myhre U, Duncan BW, Mee RB, Joshi R, Seshadri SG, HerreraVerdugo $\mathrm{O}$, et al. Apical right ventriculotomy for closure of apical ventricular septal defects. Ann Thorac Surg. 2004;78:204-8.

9. Holzer R, Balzer D, Cao QL, Lock K, Hijazi ZM, Amplatzer Muscular Ventricular Septal Defect Investigators. Device closure of muscular ventricular septal defects using the Amplatzer muscular ventricular septal defect occluder: immediate and mid-term results of a US registry. $J$ Am Coll Cardiol. 2004;43:1257-63.

10. Brizard CP, Olsson C, Wilkinson JL. New approach to multiple ventricular septal defect closure with intraoperative echocardiography and double patches sandwiching the septum. J Thorac Cardiovasc Surg. 2004;128:684-92.

11. Ootaki Y, Yamaguchi M, Yoshimura N, Oka S, Yoshida M, Hasegawa T. Surgical management of trabecular ventricular septal defects: the sandwich technique. J Thorac Cardiovasc Surg. 2003;125(3):508-12.

12. Okubo M, Benson LN, Nykanen D, Azakie A, Van Arsdell G, Coles $\mathrm{J}$, et al. Outcomes of intraoperative device closure of muscular ventricular septal defects. Ann Thorac Surg. 2001;72:416-23.

13. Bacha EA, Cao QL, Starr JP, Waight D, Ebeid MR, Hijazi ZM. Perventricular device closure of muscular ventricular septal defects on the beating heart: technique and results. J Thorac Cardiovasc Surg. 2003; 126:1718-23.

14. Bacha EA, Cao QL, Galantowicz ME, Cheatham JP, Fleishman CE, Weinstein SW, et al. Multicenter experience with perventricular device closure of muscular ventricular septal defects. Pediatr Cardiol. 2005; 26:169-75.

15. Jacobs JP, Burke RP, Quintessenza JA, Mavroudis C. Congenital Heart Surgery Nomenclature and Database Project: ventricular septal defect. Ann Thorac Surg. 2000;69(4 Suppl):S25-35.

16. Hanna B, Colan SD, Bridges ND, Mayer JE, Castaneda AR. Clinical and myocardial status after left ventriculotomy for ventricular septal defect closure. J Am Coll Cardiol. 1991;17 Suppl:110A.

17. Kleinert S, Sano T, Weintraub RG, Mee RB, Karl TR, Wilkinson JL. Anatomic features and surgical strategies in double-outlet right ventricle. Circulation. 1997;96:1233-9.

\section{Discussion}

Dr Emile A. Bacha (Boston, Mass). Alsoufi and colleagues reviewed 116 patients with mMVSDs operated on through 23 years with various techniques at The Hospital for Sick Children. A subanalysis of 27 patients who underwent a new technique, TAR, during the last 4 years was performed. Results of the TAR group were very good with regard to mortality, reoperation, incidence of heart block, and residual VSDs.

I believe that the Toronto group has developed an apparently new and effective way to treat some MVSDs. I emphasize some, because I'm just not sure that this is the panacea we're all looking for.

I have two main issues with this technique. It still is a relatively blind technique which mainly relies on probing the septum for holes, a notoriously imprecise technique. Patients with mMVSDs are one group for whom real-time imaging on the beating heart is of true help in the operating room, necessitating a shift in surgical thinking and planning.

My second concern is long-term ventricular dysfunction, which is a big problem for these patients. There is some evidence that this may be due to an intrinsic myocardial problem associated with this diagnosis. TAR has a strong trend toward longer CPB and crossclamp times. Adding prolonged myocardial ischemic times onto an intrinsically abnormal myocardium may not be the best strategy.
This brings me to the following questions. Dr Alsoufi, you failed to show any advantage in terms of residual VSDs between the TAR group and the other group. How then do you justify the longer crossclamp times associated with TAR?

The incidences of previous pulmonary arterial banding were $37 \%$ in the entire cohort and $11 \%$ in the TAR group. Have you completely abandoned primary palliation with pulmonary arterial banding, including in premature and low-birth weight babies?

Finally, when I tried a technique similar to this, I usually got leaks from underneath other trabeculated areas that were not closed because I couldn't see them, especially in the apical portion of the septum. Do you have technical points to share with us regarding how to avoid leaks at the apex?

Dr Alsoufi. I agree that aggressive probing can create VSDs in neonates. We carefully apply gentle probing, and we haven't inadvertently caused any defects in the septum so far.

Real-time imaging on a beating heart, as you suggested, is a useful tool for intraoperative localization of isolated big MVSDs. However, mMVSDs are notoriously difficult to delineate on echocardiography before the initial repair. The initial intraoperative echocardiography may reveal significant jet through large defects, however, once major defects such as large MVSDs or perimembranous VSDs are closed, additional small or moderately sized anterior and midtrabecular defects are unmasked and detected on postoperative echocardiography.

It is true that those cases require long $\mathrm{CPB}$ and crossclamp times, especially those associated with other cardiac anomalies. We have demonstrated, however, that long CPB time is no longer an adverse factor in survival in the current era. We found that those patients, as long as they leave the operating room without any residual defects and with no significant residual shunts, ventricular dysfunction, septal dysfunction or conduction problems, are able within the study limits to tolerate longer CPB time. We therefore are encouraged to spend a longer time to achieve complete septation, as long as we know we will be able to come out with no or minimal residual defects.

Two of the 3 bands that were done in the TAR group were from an outside institution. We believe that pulmonary arterial banding is not indicated for most children with mMVSDs. Pulmonary arterial bands may complicate subsequent reoperation and identification and closure of defects because of the resultant hypertrophy of the ventricular septum and right ventricular trabeculae. Obviously, there are problems with the bands such as distortion of the valve and underbanding or overbanding. Moreover, patients undergoing pulmonary arterial banding are subjected to inevitable reoperation for removal of the band and closure of remaining defects.

We have not needed to use bands in the last 4 or 5 years, although I assume that the severely sick patients, such as those with intracranial hemorrhage or very tiny kids with significant pneumonitis, are probably candidates for pulmonary arterial banding. We have not encountered such cases, however, in at least the last 5 years.

We have not shown any benefit in terms of the residual VSDs so far, even though we did propensity match and it showed that the total size of the residual shunt to be significantly lower, with a significant decrease in the number of anterior residual defects. Longer follow-up and probably larger series are needed to help us 
demonstrate whether there is any actual significant benefit in terms of residual defects. As you saw, many of those residual defects are not even evident on 2-dimensional echocardiography, they are only seen on color Doppler, and we've had no problems with 1.9 years of echocardiographic follow-up so far.

Dr Antonio F. Corno (Liverpool, UK). I respectfully disagree with all that you said about the banding. And I'm fully aware that in these meetings when we mention just "banding" it's like going back to the dinosaurs' time.

But we have a totally different policy for this group of patients with multiple VSDs thanks to the availability of the FloWatch, a telemetrically controllable device. Our protocol is that we implant the device, and then we follow up to the age of 1 year. We also have patients followed up to 2 years of age now. If needed, we can release the banding to reduce the pressure gradient with the growth of the patient. And then these patients can undergo repair at the age of 1 or even 2 years, when the body weight is 10 to $12 \mathrm{~kg}$ or more. This approach presents several advantages. First, the technical difficulty when you repair multiple VSDs at $12 \mathrm{~kg}$ is less than at 3 or $4 \mathrm{~kg}$, as with your patients.

Second, we have found that over 1 or 2 years of observation, most of the smaller defects will close, so the ischemic time required to close the residual defects is much shorter. And then, thanks to the device, we never had any distortion of the pulmonary artery, and reconstruction of the pulmonary artery has been never required. I'd like your comments on this other option.

Dr Alsoufi. Obviously our and others' past experience does not involve the adjustable band, which may eliminate many of the major problems, such as underbanding, distortion, and overbanding.

Dr Hanley's group, and others in the past, showed that preoperative banding is associated with a higher risk than primary repair. Obviously that conclusion may need to be reassessed with the adjustable bands. We don't have those in Toronto, unfortunately.

The other important fact is that almost half of the patients will require early operation for the associated cardiac anomalies rather than the VSDs. In those cases, we prefer to leave the operating room with no residual lesions, because the patients will do better postoperatively. Our indication for operation on patients with isolated mMVSDs is heart failure. Although I agree with you that surgery is probably easier and better tolerated in an older patient, our experience with primary repair at an early age proves that this is a safe treatment alternative.

Dr Carlos J. Troconis (Caracas, Venezuela). One of my major concerns is the transoperative identification of these multiple VSDs. To address this issue, we have been using as well as per the apical VSDs, the cardioscope to identify those holes within the trabeculae either directly or by instilling cold saline solution into the left ventricular trough out an apical left ventricular vent, or even threading a small balloon from the left ventricular side. Do you think this technique will assist you in identifying the multiple defects and allow you to be more precise and accurate in their closure, as well as shortening the anatomic evaluation, the surgical closure expenditure, and the crossclamp and pump times? Not leaving residual defects or heart block with good ventricular function is the ultimate goal.
Dr Alsoufi. It's definitely an elegant technique, and I'm sure it has a role in some cases. In fact, Dr Black from our hospital in Toronto has reported his experience with the use of cardioscopy to close MVSDs in the past. We have not needed to use it in the last few years, because we have used the TAR technique. Maybe because we're not putting a patch, we don't have to delineate the exact edges of the defect; rather, we need to identify what trabeculations will occlude those exit points. Nonetheless, I'm sure cardioscopy is a useful tool that the surgeon can apply in some circumstances.

Dr Christo I. Tchervenkov (Montreal, Quebec, Canada). I would like to raise a couple of other issues. How do you count the number of VSDs? We all very well know that when you look at the VSD, or when you look at the left ventricular aspect of the left ventricular septum, what is a single VSD, as viewed from the right side, with all the crossing trabeculations, may look like 5 VSDs. It sounds impressive to say, "I closed 9 VSDs in a patient," when in reality it is 1 dominant VSD and perhaps a second VSD with many trabeculations. So that's one issue.

The second issue, the pitfall of this technique, which is excellent and it should definitely be in the armamentarium, is to perhaps mislead some surgeons to go off to really small VSDs in the muscular septum or the apical septum that should not really be addressed because they're going to close spontaneously. So how do you decide the relative contribution of the small midmuscular or apical MVSDs in addition to perhaps dominant perimembranous or outlet VSDs? I mean, that is the real key issue.

Dr Alsoufi. The definition of Swiss cheese septum is pretty vague. In the last Society of Thoracic Surgeons nomenclature, the septum was defined Swiss cheese if 4 or more VSDs existed. We often find that echocardiography underestimates the number of VSDs, and most of the times we find more probe patent VSDs that we have to close.

We aim to leave the operating room with no residual shunt. I'm not sure we can tell whether or not the VSD that will close is going to contribute to the patient's status at the end. Often you close a big perimembranous VSD, and once you relieve that big shunt, suddenly another small defect that you thought was not significant becomes significant and starts causing problems, and you have to reoperate. Therefore our goal usually is to eliminate any interventricular communication.

The other question was regarding the number of VSDs closed. The number obviously depends on the surgical technique. So if you're going to cut the trabeculations and identify the edges of the defect and close it with a patch, you are going to end up saying that I closed 1 VSD. We don't like to cut the trabeculations, because they may cause problems to the septum function, so we are targeting the exit points on the right side. So 1 single VSD that would be closed with a patch may be 3 or 4 exit points requiring TAR sutures. I don't think it matters how many VSDs you've closed or you started with. What matters is your end product. We believe that the surgeon's goal should be, again, no residual defects with good function and no heart block. 
TABLE E1. Variables used to construct the propensity score (area under the curve 0.88)

\begin{tabular}{ll}
\hline Variable & $\boldsymbol{P}$ value \\
\hline Tetralogy of Fallot & .09 \\
Morphologic VSD type & \\
$\quad$ Inlet & .004 \\
Midmuscular & .01 \\
$\quad$ Anterior & .004 \\
Surgeon & \\
A & .005 \\
B & .08 \\
Aortic crossclamp time (min) & .15 \\
\hline
\end{tabular}

VSD, ventricular septal defect.
TABLE E2. Clinical profile of all patients with multiple muscular ventricular septal defects (mMVSDs)

\begin{tabular}{lc}
\hline No. of patients & 116 \\
Age range & $3 \mathrm{~d}-18.1 \mathrm{y}$ \\
Median age & $9 \mathrm{mo}$ \\
Male sex (No.) & $47(41 \%)$ \\
Previous pulmonary arterial band (No.) & $46(40 \%)$ \\
Isolated mMVSDs (No.) & $52(45 \%)$ \\
Associated cardiac anomalies (No.) & $64(55 \%)$ \\
Coarctation of the aorta & $25(22 \%)$ \\
Transposition of the great arteries & $13(11 \%)$ \\
Atrioventricular septal defect & $8(7 \%)$ \\
Tetralogy of Fallot & $7(6 \%)$ \\
Double-outlet right ventricle & $4(4 \%)$ \\
Persistent truncus arteriosus & $2(2 \%)$ \\
Other & $5(4 \%)$ \\
\hline
\end{tabular}

TABLE E3. Multivariable regression models for operative mortality, pacemaker implantation, and time-related survival for 116 patients with multiple muscular ventricular septal defects

\begin{tabular}{|c|c|c|c|c|c|}
\hline Variable & Parameter estimate & SE & OR & $95 \% \mathrm{Cl}$ & $P$ value \\
\hline \multicolumn{6}{|c|}{$\begin{array}{l}\text { Multivariable logistic regression model } \\
\text { for operative mortality }\end{array}$} \\
\hline DORV & 3.80 & 1.28 & 44.71 & $3.6-548.9$ & .003 \\
\hline Ventriculotomy & 1.85 & 0.76 & 6.4 & $1.2-34.5$ & .03 \\
\hline Fewer VSDs repaired & 1.54 & 0.76 & 4.7/per VSD & $1.1-20.4$ & .04 \\
\hline \multicolumn{6}{|c|}{$\begin{array}{l}\text { Multivariable logistic regression model } \\
\text { for pacemaker implantation }\end{array}$} \\
\hline DORV & 2.28 & 1.05 & 9.7 & 1.2-76.5 & .03 \\
\hline Postoperative PAB & 1.87 & 0.97 & 6.8 & $0.96-43.4$ & .055 \\
\hline \multicolumn{6}{|c|}{$\begin{array}{l}\text { Cox proportional hazard regression model } \\
\text { for time-related death }\end{array}$} \\
\hline DORV & 2.11 & 0.91 & 8.27 & $1.39-49.3$ & .02 \\
\hline Longer CPB time & 0.02 & 0.007 & $1.02 / \mathrm{min}$ & $1.003-1.03$ & .02 \\
\hline \multicolumn{6}{|c|}{ Interaction between CPB time and era $3^{*}$} \\
\hline & $! 0.010$ & 0.004 & 0.99 & $0.982-0.998$ & .04 \\
\hline
\end{tabular}

$S E$ standard error, ; $O R$, Odds ratio; $C l$, confidence interval; $D O R V$, double-outlet right ventricle; $V S D$, ventricular septal defect; $P A B$, pulmonary arterial banding; $C P B$, cardiopulmonary bypass. *Longer bypass time has been neutralized as a risk factor for death in era 3. 


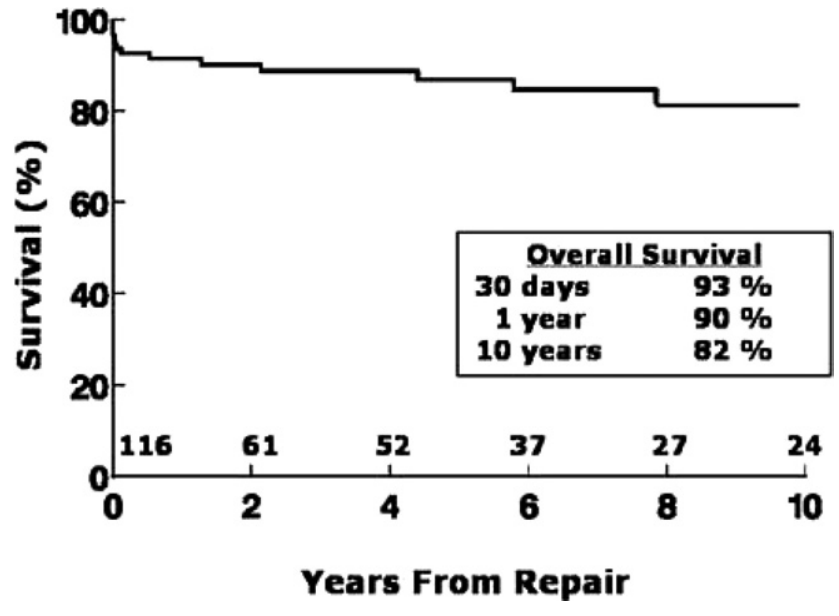

Figure E1. Kaplan-Meier estimated survival after repair of multiple muscular ventricular septal defects for entire cohort of 116 patients.

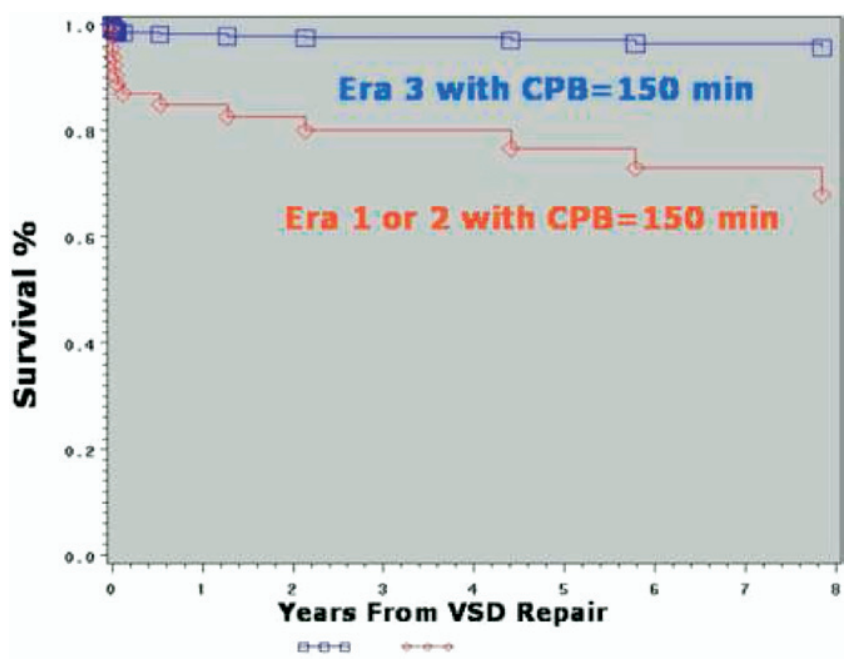

Figure E2. Risk-adjusted predictions for time-related survival after repair of multiple ventricular septal defects (VSDs) stratified by era of operation. There was significant interaction term whereupon similar of cardiopulmonary bypass (CPB) duration had been neutralized in most recent era. Longer CPB time was a risk-factor in earlier eras, but not within most recent era (Era 3).
TABLE E4. Clinical profile of patients with multiple muscular ventricular septal defects (mMVSDs) undergoing transatrial re-endocardialization

\begin{tabular}{lc}
\hline No. of patients & 27 \\
Age (range) & $15 \mathrm{~d}-7.2 \mathrm{y}$ \\
Age (median) & $196 \mathrm{~d}$ \\
Weight (kg, median and range) & $4.9 \mathrm{~kg}(2.4-17.6 \mathrm{~kg})$ \\
Body surface area (median and range) & $0.3(0.17-0.66)$ \\
Male sex (No.) & $7(26 \%)$ \\
Isolated mMVSDs (No.) & $14(52 \%)$ \\
Associated cardiac anomalies (No.) & $13(48 \%)$ \\
Coarctation of the aorta & $5(19 \%)$ \\
Atrioventricular septal defect & $3(11 \%)$ \\
Tetralogy of Fallot & $3(11 \%)$ \\
Transposition of the great arteries & $1(4 \%)$ \\
Double-outlet right ventricle & $1(4 \%)$ \\
\hline
\end{tabular}

